\title{
Experimental testing of Timoshenko predictions of supercritical natural frequencies and mode shapes for free-free beams
}

Brøns, Marie; Thomsen, Jon Juel

Published in:

Journal of Sound and Vibration

Link to article, DOI:

10.1016/j.jsv.2019.114856

Publication date:

2019

Document Version

Peer reviewed version

Link back to DTU Orbit

Citation (APA):

Brøns, M., \& Thomsen, J. J. (2019). Experimental testing of Timoshenko predictions of supercritical natural frequencies and mode shapes for free-free beams. Journal of Sound and Vibration, 459, [114856].

https://doi.org/10.1016/j.jsv.2019.114856

\section{General rights}

Copyright and moral rights for the publications made accessible in the public portal are retained by the authors and/or other copyright owners and it is a condition of accessing publications that users recognise and abide by the legal requirements associated with these rights.

- Users may download and print one copy of any publication from the public portal for the purpose of private study or research.

- You may not further distribute the material or use it for any profit-making activity or commercial gain

- You may freely distribute the URL identifying the publication in the public portal

If you believe that this document breaches copyright please contact us providing details, and we will remove access to the work immediately and investigate your claim 


\title{
Experimental testing of Timoshenko predictions of supercritical natural frequencies and mode shapes for free-free beams
}

\author{
Marie Brøns*1, Jon Juel Thomsen ${ }^{1}$
}

\begin{abstract}
In this experimental work a scanning laser Doppler vibrometer is used to measure mode shapes and natural frequencies of beam bending vibrations above the critical frequency predicted by Timoshenko theory. Above the critical frequency the mode shapes are intricate, so to quantitative compare theoretical and experimental mode shapes, the modal assurance criterion (MAC) is applied. This reveals that the order of the modes is not as theory predicts, and that other plane modes can easily be misinterpreted as bending modes. This can lead to an incorrect comparison of natural frequencies. With the MAC tool it is found that the correlation between theoretical mode shapes and measured mode shapes is very high; It can be concluded that supercritical Timoshenko-predicted mode shapes agrees well with the experimentally observed modes, and the supercritical natural frequencies deviate from Timoshenko theory within five percent. Comparisons are made to other studies, and against a numerical 3D model.
\end{abstract}

Keywords: Timoshenko beam theory, mode shapes, modal assurance criterion, critical frequency, shear bending, rotary inertia, scanning laser Doppler vibrometry, Timoshenko frequency pairs

\section{Introduction}

Beam vibrations and accuracy of different beam models have been studied extensively over the last century - Timoshenko beam theory (TBT) especially [1]. The well established theory is a refinement of the classical Bernoulli-Euler theory, as it takes shear deformation and rotary inertia into account [2]. It is suitable to use even for short beams, and for higher vibration modes, where the wavelength approaches the thickness of the beam. Timoshenko theory predicts a critical frequency $f_{\mathrm{c}}$ beyond which the natural frequencies come in close pairs. In this work these will be referred to as supercritical natural frequencies and mode shapes, as in [3]. The theory's predictions above the critical frequency for different boundary conditions have long been subject to debate. Distinguishing between boundary conditions is important, as the TBT equations factorizes for a simple supported beam (and guided-hinged or guided-guided [4]), whereas they do not for a free-free beam. There are only few experimental contributions to support or reject the Timoshenko theory predictions above the critical frequency. Limitations in measurement equipment have previously made it difficult to measure a sufficient number of points along a beam length to capture the higher mode shapes, and furthermore it is difficult to excite the higher modes of structures at sufficient strength for good measurements. Both factors makes it hard to study modes above the critical frequency. By use of a scanning laser Doppler

\footnotetext{
${ }^{1}$ Technical University of Denmark, Department of Mechanical Engineering, *maribr@mek.dtu.dk
} 
vibrometer (SLDV), this work experimentally measures mode shapes and natural frequencies for a free-free beam also above the critical frequency. The SLDV can measure vibrations along the beam length at high resolution (this study uses 160 points), so the setup makes it possible to identify the accuracy of TBT also above the critical frequency. Proper validation of Timoshenko theory predictions for free-free boundary conditions above the critical frequency requires that both natural frequencies and mode shapes agrees reasonably with experimental results.

Previous reports of experimental studies have reached opposing conclusions. In 2002 Chan et al. [3] reported an experiment with an accelerometer on a free-free beam, excited by a shaker. The experiment showed agreement between measured frequencies and TBT predictions, also beyond $f_{\mathrm{c}}$. The measurements captured the first two sets of frequency pairs. The mode shapes belonging to these pairs showed less conclusive results, as the experiment only consisted of 11 measurement points along the beam length, but they show nothing that directly contradicts TBT predictions.

In 2006 Stephen and Puchegger [5] reported on the valid range of TBT for free-free boundary conditions by an experimental approach. The experiment was performed on a very short and thick aluminium block (almost symmetrical in the two transverse planes), having dimensions that only give four natural frequencies below $f_{\mathrm{c}}$. Measurements were made in both transverse planes, but only of natural frequencies, not mode shapes. Although the work does not mention it, the experiment showed that the frequencies come in pairs. Due to deviations between TBT predictions and the measured frequencies, the authors conclude that TBT is unreliable above $f_{\mathrm{c}}$, and that such predictions should be disregarded. Nonetheless, scrutinizing the results for both transverse planes, the first two supercritical frequency pairs deviate less than $4 \%$ from TBT predictions. This could also be considered a small error as these modes are actually $18 \%$ beyond the critical frequency, and the wavelength in these cases are approaching the beam thickness.

In recent studies by Diaz. et al. [6, 7] a free-free aluminium beam is tested. The measurements are made with a novel setup, with an electromagnetic acoustic exciter on the beam that moves along the beam length. Both the natural frequencies and mode shapes are measured up to $47 \mathrm{kHz}$, capturing six supercritical frequency pairs. In the study only a qualitative comparison of mode shapes are made, with the experimental mode shapes depicted atop the theoretical. The conclusion is, as in [3], that TBT is an excellent theory, even above $f_{\mathrm{c}}$.

Concurrent with these experimental observations, work of Elishakoff et al. [1] concludes that "Timoshenko's theory gives only a rough approximation to the aforementioned second spectrum", and suggests instead a modified version, where there is no so-called second spectrum. The term second spectrum is a more helpful description for the simply supported than for the free-free beam, as the equation factorizes, giving two distinct spectra beyond $f_{\mathrm{c}}$. In the case of the free-free beam there is only one equation, giving pairs beyond $f_{\mathrm{c}}$, but these are not distinctly separated. However, the second spectrum can in this case be defined as one of the frequencies in a pair. It has also been a long-standing debate whether or not a second spectrum even exists, and if it does, if it is physically meaningful [8, 9, 10]. Here, quoting [1]: "Timoshenko's (1921) theory predicts two frequency spectra [..]. This is an undisputed fact", and [1] also refers to the experimental work in [3]. Due to this, and because of the experimental observations mentioned above, the existence of a second spectrum (and thus paired frequencies) is not further questioned in this work, but as there is no factorization of equations for the free-free beam, the term second spectrum is also not applied further.

Summing up, it appears evident that the validity and interpretation of TBT beyond the critical frequency is a complicated subject. The current debate could roughly be divided into three 
positions: 1) Supercritical TBT predictions are physically meaningless and should be disregarded [4, 5]; 2) Supercritical predictions are rough and inaccurate and should be disregarded (and possibly the equation should be modified [1]); and 3) Supercritical predictions are accurate and should not be disregarded [6, 7]. This present work focus on testing further the last two positions, by experimentally exciting a beam also beyond the critical frequency to test the accuracy of TBT predicted frequency pairs and their corresponding mode shapes.

For subsequent reference, Section 2 briefly outlines the Timoshenko theory for free-free boundary conditions, and also shows the theoretical effect of shear and rotary inertia on the mode shapes above the critical frequency. Section 3 describes the experimental setup and Section 4 the experimental results, while Section 5 discusses the results and relates these to other work. Section 6 concludes on the knowledge obtained from the new experiments.

\section{Timoshenko theory - A brief overview of equations, and the influence of shear and rotation above the critical frequency}

Timoshenko's differential equation describing transverse displacement $y(x, t)$ of an initially straight beam is given by [2]:

$$
E I \frac{\partial^{4} y}{\partial x^{4}}+\rho A \frac{\partial^{2} y}{\partial t^{2}}-\rho I\left(1+\frac{E}{k G}\right) \frac{\partial^{4} y}{\partial x^{2} \partial t^{2}}+\frac{\rho^{2} I}{k G} \frac{\partial^{4} y}{\partial t^{4}}=0
$$

where $E I$ is the flexural stiffness, $\rho A$ the mass per unit beam length, $x$ the longitudinal coordinate, $t$ time, $\rho I$ the mass moment of inertia, $k$ the shear coefficient, and $G$ the modulus of rigidity. The single equation arise from two coupled differential equations that can be derived using Figure 1. which shows a differential beam element deformed in bending and shear, in absence of normal forces, where $T(x, t)$ are the inner shear forces and inner $M(x, t)$ the bending moments. The angle $\Psi$ is between the normal to the deformed cross section and the $x$-axis, while the angle $\Gamma$ is between the normal to the deformed cross section and tangent to the beam axis. This means that the actual rotation of a material cross section in the inertial $(x, y)$-coordinate system is $\Psi$, and thus the associated inertial forces are $\rho I \ddot{\Psi}$. When $\Gamma$ is zero the cross section is normal to the beam axis; this is the case of pure bending, i.e. with no shear, and $\frac{\partial y}{\partial x}=\Psi$.

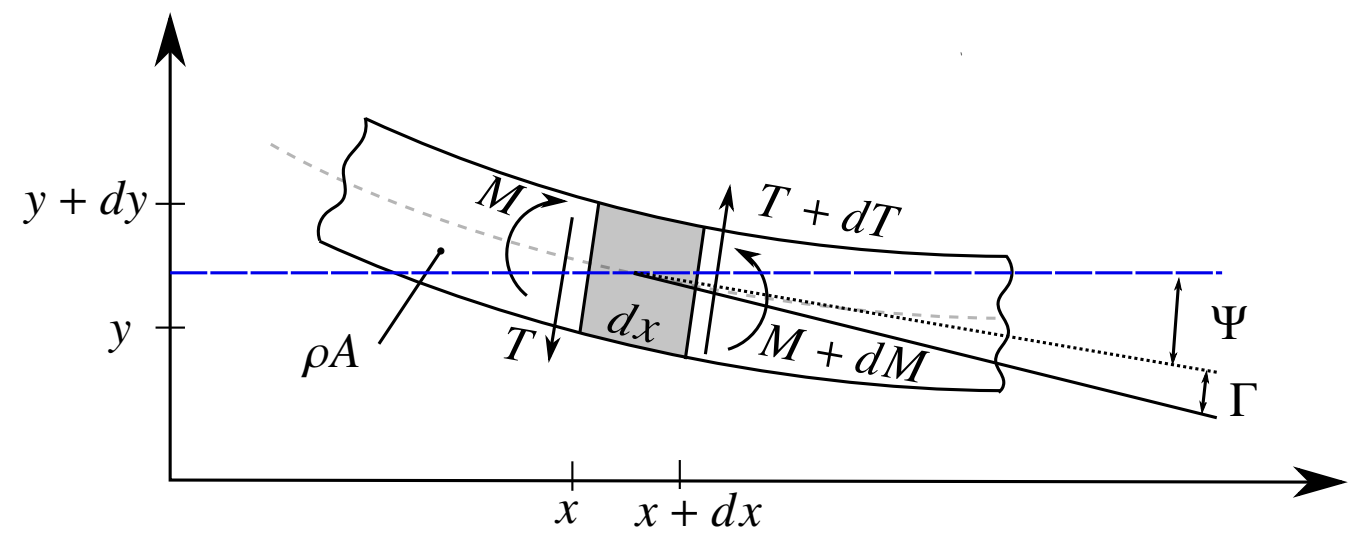

Figure 1: Differential beam element. Short dashed line: Deformed beam axis; long dashed line (blue): parallel to the $x$-axis; dotted line: normal to deformed cross section; solid line: tangent to beam axis. (Adapted from [2]) 
With non-zero $\Gamma$, Figure 1 shows, for small deflection the slope of $y$ is the sum of the two angles $\Psi$ and $\Gamma$ :

$$
\frac{\partial y}{\partial x}=\Psi+\Gamma .
$$

The two coupled partial differential equations describing the transverse displacement $y(x, t)$ and the cross section rotation $\Psi(x, t)$ can be setup by applying Newton's second law for the $y$-direction of Figure 1, and likewise take the moment around the center of mass, giving:

$$
\begin{gathered}
\frac{\partial}{\partial x}\left[E I \frac{\partial \Psi}{\partial x}\right]+k\left(\frac{\partial y}{\partial x}-\Psi\right) A G=\rho I \frac{\partial^{2} \Psi}{\partial t^{2}}, \\
\frac{\partial}{\partial x}\left[k\left(\frac{\partial y}{\partial x}-\Psi\right) A G\right]=\rho A \frac{\partial^{2} y}{\partial t^{2}}
\end{gathered}
$$

as the shear force is given by $T=k\left(\frac{\partial y}{\partial x}-\Psi\right) A G$ and the elastic moment by $M=E I \frac{\partial \Psi}{\partial x}$ [2]. In the case of harmonic vibrations, the solution to the coupled equations (3) can be written as:

$$
y(x, t)=\varphi(x) \sin (\omega t+\varepsilon), \quad \Psi(x, t)=\psi(x) \sin (\omega t+\varepsilon), \quad \Gamma(x, t)=\gamma(x) \sin (\omega t+\varepsilon)
$$

where $\varphi(x), \psi(x)$ and $\gamma(x)$ are eigenfunctions, describing the transverse displacement, rotation and shear angle amplitude, respectively, and $\omega$ the oscillation frequency. Substituting (4) into (3) gives two ordinary differential equations for TBT:

$$
\begin{gathered}
\psi^{\prime \prime}(x)+\left(\frac{\omega^{2}}{c_{0}^{2}}-\frac{k G}{E r^{2}}\right) \psi(x)+\frac{k G}{E r^{2}} \varphi^{\prime}(x)=0, \\
\varphi^{\prime \prime}(x)+\frac{E}{k G} \frac{\omega^{2}}{c_{0}^{2}} \varphi(x)-\psi^{\prime}(x)=0,
\end{gathered}
$$

where $r^{2}=I / A$ and $c_{0}^{2}=E / \rho$, and primes denote differentiated w.r.t. $x$. The solution for $\varphi(x)$ has the form [11]:

$$
\varphi(x)=C_{1} \cosh \alpha \frac{x}{l}+C_{2} \sinh \alpha \frac{x}{l}+C_{3} \cos \beta \frac{x}{l}+C_{4} \sin \beta \frac{x}{l},
$$

and the associated $\psi(x)$, can be expressed by the same constants as:

$$
\psi(x)=\frac{(E / k G) p^{2}+\alpha^{2}}{\alpha l}\left(C_{1} \sinh \alpha \frac{x}{l}+C_{2} \cosh \alpha \frac{x}{l}\right)+\frac{(E / k G) p^{2}-\beta^{2}}{\beta l}\left(C_{3} \sin \beta \frac{x}{l}-C_{4} \cos \beta \frac{x}{l}\right)
$$

with dimensionless parameters $p, \alpha$, and $\beta$ :

$$
\begin{aligned}
& p^{2}=\left(\omega l / c_{0}\right)^{2}, \\
& \left.\begin{array}{l}
\alpha^{2} \\
\beta^{2}
\end{array}\right\}=\frac{p^{2}}{2}\left[\mp\left(1+\frac{E}{k G}\right)+\sqrt{\left(1-\frac{E}{k G}\right)^{2}+\left(\frac{2 l}{p r}\right)^{2}}\right] .
\end{aligned}
$$

Note the presence of $E / k G$ in (7), which is incorrectly swapped around in [11]; this error has no influence on the first modes, but becomes pronounced for higher modes. In (9) there is a 
plus sign in front of all terms defining $\beta^{2}$, and as all parameters are positive $\beta^{2}$ must thus be a real and positive for all values of $p$. For $\alpha$ this is not the case: The higher the frequency $\omega$, the smaller the contribution from the term $(2 l / p r)^{2}$ in $(9)$, so that for a certain $\omega, \alpha$ will become an imaginary number. In particular:

$$
\alpha \rightarrow \pm i \sqrt{\frac{E}{k G}} p, \text { for } p \rightarrow \infty
$$

where $i$ is the imaginary unit. The corresponding value of $\omega$, where $\alpha$ switches from real to imaginary, is called the critical frequency $\omega_{c}$; it occurs when the radical in (9) becomes negative. The critical frequency can thus be calculated by zeroing (9), and by inserting (8) and $r^{2}=I / A$ :

$$
\omega_{c}=\sqrt{\frac{G k A}{\rho I}} .
$$

There are two cases $\omega<\omega_{c}$ and $\omega>\omega_{c}$, the second case will subsequently be referred to as supercritical (natural) frequencies.

\subsection{Frequency equation for free-free boundary conditions}

The boundary conditions for the beam ends are, with $x=0$ at the beam midpoint [11]:

$$
\varphi^{\prime}( \pm l / 2)-\psi( \pm l / 2)=\psi^{\prime}( \pm l / 2)=0
$$

and the geometric conditions at the midpoint are [11]:

$$
\begin{aligned}
\text { for symmetric (even) modes } & \psi(0)=\varphi^{\prime}(0)-\psi(0)=0, \\
\text { for antisymmetric (odd) modes } & \varphi(0)=\psi^{\prime}(0)=0,
\end{aligned}
$$

since for symmetric modes $T(0)=0$, and the tangent to the beam axis is zero at $x=0$, while for antisymmetric modes $M(0)=0$ and the deflection is zero at $x=0$. The additional conditions in (13) are redundant; they just express conditions of continuity and symmetry already present in the problem formulation (e.g. it follows by mere inspection of (5) that all modes must be either symmetric or antisymmetric w.r.t. $x=0$ ). However, imposing these conditions explicitly splits up the frequency equation into two much simpler equations, for even and odd modes respectively.

Applying (12)-(13) to the TBT eigenfunctions (6)-(7) gives four linear homogeneous algebraic equations in the unknown constants $C_{1}, C_{2}, C_{3}, C_{4}$. The TBT frequency equation is obtained by zeroing the determinant of the coefficient matrix of this system giving, for the symmetric and antisymmetric modes, respectively:

$$
\begin{aligned}
& \tan \frac{\beta}{2}+\frac{\beta}{\alpha} \frac{\beta^{2}-(E / k G) p^{2}}{\alpha^{2}+(E / k G) p^{2}} \tanh \frac{\alpha}{2}=0, \\
& \tan \frac{\beta}{2}-\frac{\alpha}{\beta} \frac{\alpha^{2}+(E / k G) p^{2}}{\beta^{2}-(E / k G) p^{2}} \tanh \frac{\alpha}{2}=0,
\end{aligned}
$$

which can be solved for $\omega$ numerically. The corresponding solutions for the constants $C_{1}, C_{2}, C_{3}, C_{4}$ are found (except for a common arbitrary factor) solving the above mentioned algebraic equations with (14) inserted. Figure 2 depicts the left-hand sides $g$ of frequency equations (14) as 
functions of $\omega$, for odd and even modes. The physical parameters are set to that of the experimental beam (cf. Section 3), with the critical frequency $f_{\mathrm{c}}=17.04 \mathrm{kHz}$. Natural frequencies are characterized by zeroes of $g$. Up until the critical frequency the natural frequencies alternate between odd and even modes, as the solid and dashed line intercept zero in turn. Above $f_{\mathrm{c}}$ the odd and even modes switch order, and are very close together, as pairs. This switching of modes continues, in a seemingly unsystematic way, also higher up in the spectrum than shown in the figure. The order of the mode odd/even mode pairs is very sensitive to changes in parameters, e.g. changing the area moment of inertia $I$ will change the coupling between rotation and bending (cf. (5a) ) which can change the order. Likewise, the order can change when changing the shear coefficient $k$ and the length $l$. The behaviour is unique to TBT, whereas the modified equation suggested in [1] does not have this transition and the pairs, but continues to alternate between odd and even modes, roughly evenly distributed.

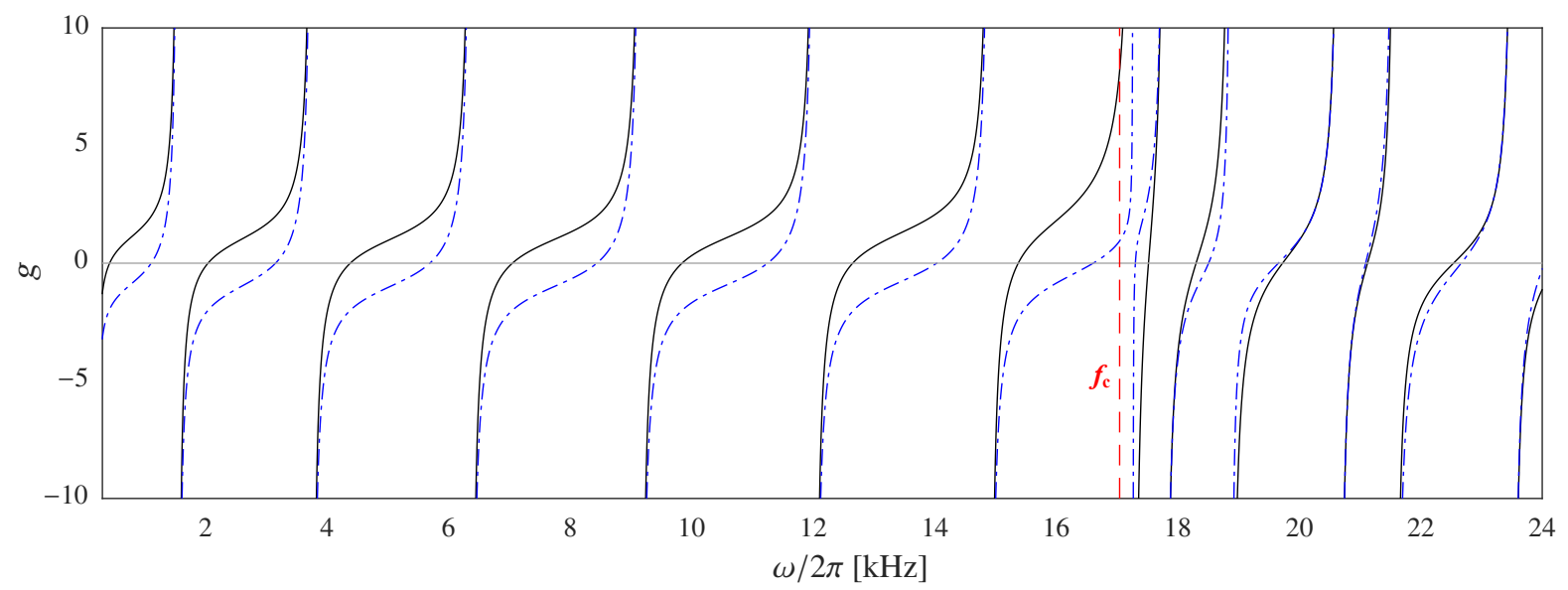

Figure 2: Left-hand sides $g(\omega)$ of the frequency equations (14) (physical parameters as for the experimental beam, cf. Section 3). Solid line: even modes; dash-dotted line: odd modes; dashed line: critical frequency.

\subsection{Theoretical predictions of shear and rotation of the experimental beam}

Considering only Figure 2 it seems at first hand odd how the frequencies suddenly occur in pairs. Intuitively, beyond the critical frequency it should take 'energy', i.e. a shift in frequency, to go up a mode (half a wavelength), so how is it possible that two independent modes be so close in frequency? Figure 3 suggests an explanation to the pairs: Each pair actually consists of two very different deformation compositions of shear and rotation, but the sum is alike, resulting in frequencies close together.

Elaborating on this Figure 3, it shows $\varphi^{\prime}(x)$, the slope of the transverse vibrations, and its two components: the shear angle $\gamma(x)$ and the cross section rotation $\psi(x)$ (from (4) and (2) it follows $\varphi^{\prime}(x)=\psi(x)+\gamma(x)$ ). The physical parameters are as for the experimental beam (cf. Section 3). Below $f_{\mathrm{c}}$ the slope of the deflection $\varphi^{\prime}$ is primarily due to rotation $\psi$ of the cross section. The shear angle $\gamma$ has an increasing influence when approaching $f_{\mathrm{c}}$, but it is still in phase with $\psi$. Above $f_{\mathrm{c}}$ this changes, so that $\psi$ and $\gamma$ are of the same magnitude but out of phase. A smaller the slenderness ratio would mean that the shearing would influence at even lower modes (i.e. a lower critical frequency).

This behaviour has been partly reported in [8] (showing a single mode of shear and rotation, but with $\varphi(x)$ instead of $\varphi^{\prime}(x)$ ). There are also examples where $\psi(x)$ is plotted, without the shear angle [12, 13], and most recently in [14] $\psi, \gamma$ and $\varphi^{\prime}$ are plotted in a row of figures for selected 
modes, but to the best of the authors knowledge graphs of the components $\psi$ and $\gamma$ and the sum $\varphi^{\prime}$ together, for modes both below and beyond the critical frequency, have not been presented before.

$\# 1$

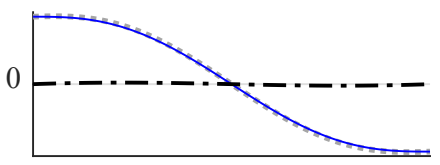

\#4

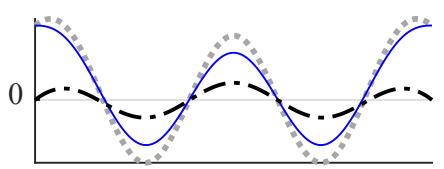

\#7

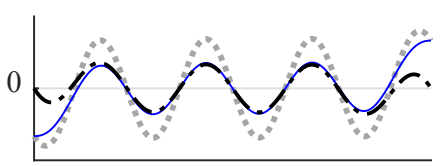

\#10

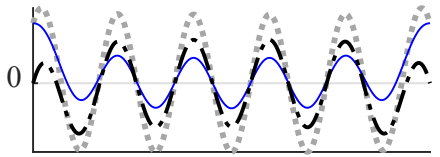

\#13

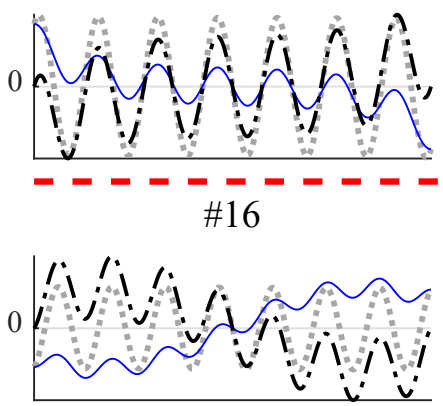

\#19

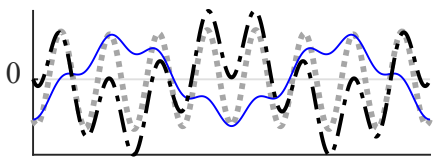

\#22

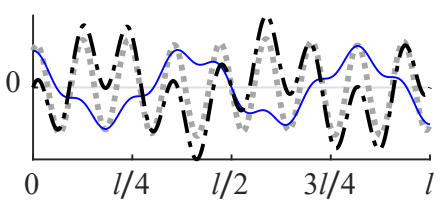

\#2

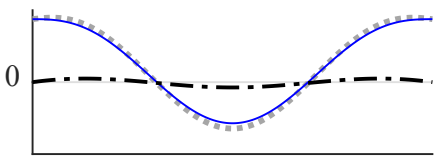

\#5

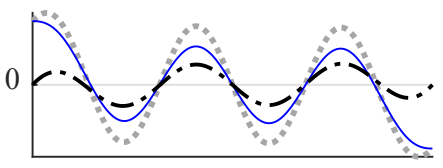

\#8

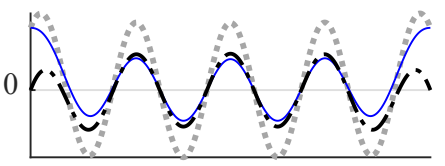

\#11
\#3

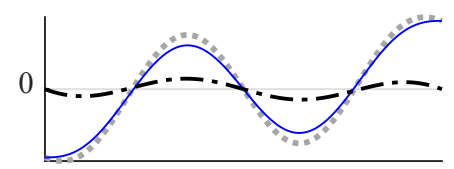

\#6

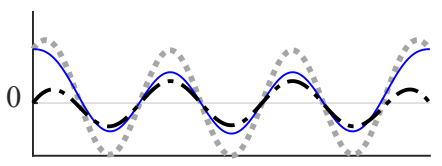

\#9

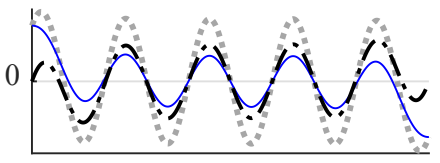

\#12

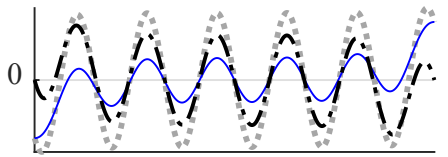

$\# 14 \omega<\omega_{\mathbf{c}}$

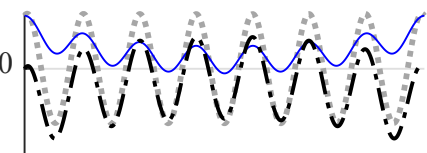

$\# 17$

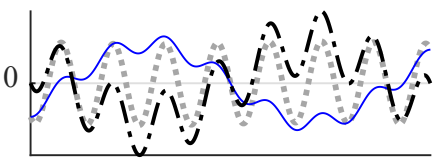

\#20

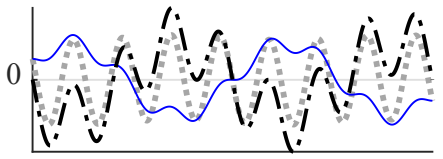

\#23

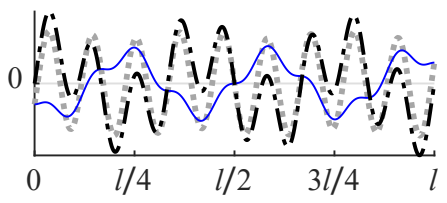

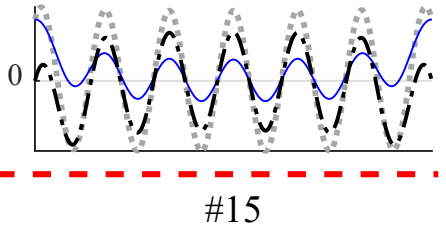

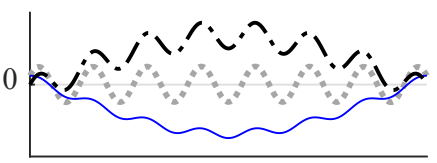

\#18

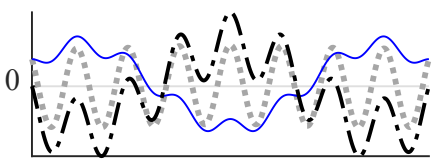

\#21

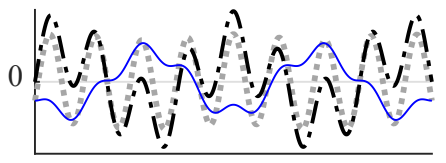

\#24

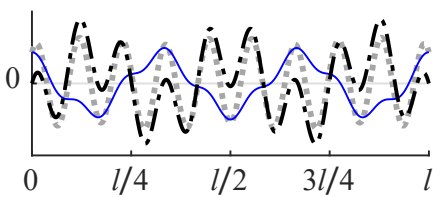

Figure 3: TBT predictions of the slope of mode shapes and their components for the first 24 modes of the free-free experimental beam: Solid line (blue): cross-section rotation $\psi$; dashed line (black): shear angle $\gamma$; dotted line (grey): deflection slope $\varphi^{\prime}=\psi+\gamma$. 


\subsection{Using the Modal Assurance Criterion for matching mode shapes}

Graphically overlaid mode shapes only allow for qualitative comparison. A way of quantitatively comparing mode shapes is to compute the Modal Assurance Criterion-value (MAC) between two modes. The mode shapes $\varphi_{m}$ are then considered vectorial quantities, i.e. discretized continuous mode shapes, or experimental mode shapes measured pointwise. The MAC-value for two mode shapes $\varphi_{m}$ and $\varphi_{n}$ is [15]:

$$
\operatorname{MAC}\left(\boldsymbol{\varphi}_{m}, \boldsymbol{\varphi}_{n}\right)=\frac{\left|\boldsymbol{\varphi}_{m}^{T} \boldsymbol{\varphi}_{n}\right|^{2}}{\boldsymbol{\varphi}_{m}^{T} \boldsymbol{\varphi}_{m} \boldsymbol{\varphi}_{n}^{T} \boldsymbol{\varphi}_{n}},
$$

which for every $m, n$ produces a single scalar value between zero (for orthogonal mode shapes) and unity (for identical mode shapes).

Actually, since the directly measurable part $\varphi$ of a mode in itself do not constitute a full normal mode (the $\boldsymbol{\psi}$-components are missing), different modes $\boldsymbol{\varphi}_{m}$ and $\boldsymbol{\varphi}_{n}$ will not generally produce a zero MAC-value. Still, with the $\varphi$-components containing most of what describes a measurable mode (for the lowest modes almost exclusively), the calculated patterns of MACvalues based on just $\varphi$ provides for a quantitative comparison and proper matching of theoretically/numerically calculated and experimentally measured modes.

According to [15] it is difficult to set a fixed minimum value MAC should have to indicate good correlation between modes; generally $\mathrm{MAC}=0.9$ is considered a good correlation, when comparing experiments with theory [15]. The MAC can only provide indications of consistency between a set of modes, and not any measure of modal orthogonality (due to the absence of a mass matrix [16]). A MAC-value close to unity can indicate good correlation, but can also reflect too few measuring points. This is not a problem for the experiments in this work, due to the ability to measure at high spatial resolution with the SLDV. The exact MAC-values should not be attached with too much importance, but are good for ensuring that theoretical and experimental modes have been matched correctly, with no consideration to possible nearness in corresponding natural frequencies, and with no sensitivity to possible unmeasured modes.

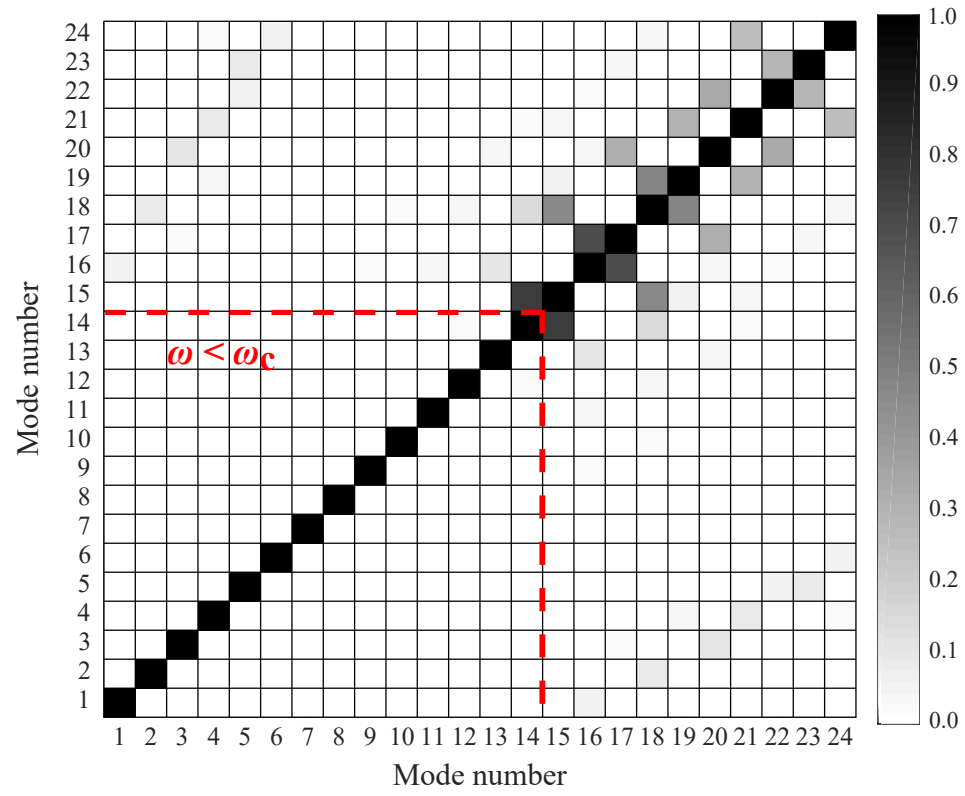

Figure 4: MAC-matrix for the first 24 beam modes predicted by TBT. 
For illustration Figure 4 shows the MAC-matrix for discretized versions of $\varphi(x)$ as predicted by TBT for the first 24 modes compared to themselves (again the physical parameters are set to that of the experimental beam, cf. Section 3). Below the critical frequency (cf. red dashed line) all modes are matched correctly if the MAC-matrix has a diagonal of ones (black), and zeros (white) in the off-diagonal elements. Above $f_{\mathrm{c}}$ the MAC-matrix forms a sort of chess pattern, with unit diagonal values (black), and less than unity off-diagonal values (grey), as the mode pairs contain some elements of each other; this reflects the complex compositions of shear and rotation (cf. Figure 3). The MAC tool can help match the mode shapes, as a similar pattern should appear when comparing experimentally measured modes with TBT modes, if TBT provides a reasonable prediction of mode shapes.

\section{Experimental setup}

Figure 5 shows the lab setup, which consists of a beam suspended in elastic bands: soft enough to provide effectively free-free boundary conditions even for the lowest mode, a PSV 500 scanning laser vibrometer pointing down on the width of the beam, and a B\&K shaker type 4809 attached with a stinger below the beam. The shaker is connected to a PSV computer, from where the input signal is controlled. A pseudo-random excitation signal is applied in the frequency range of interest $(0-22 \mathrm{kHz})$. Reflective tape (providing ignorable damping, mass and stiffness) is mounted to the beam top along its length to improve the quality of the laser measurements.

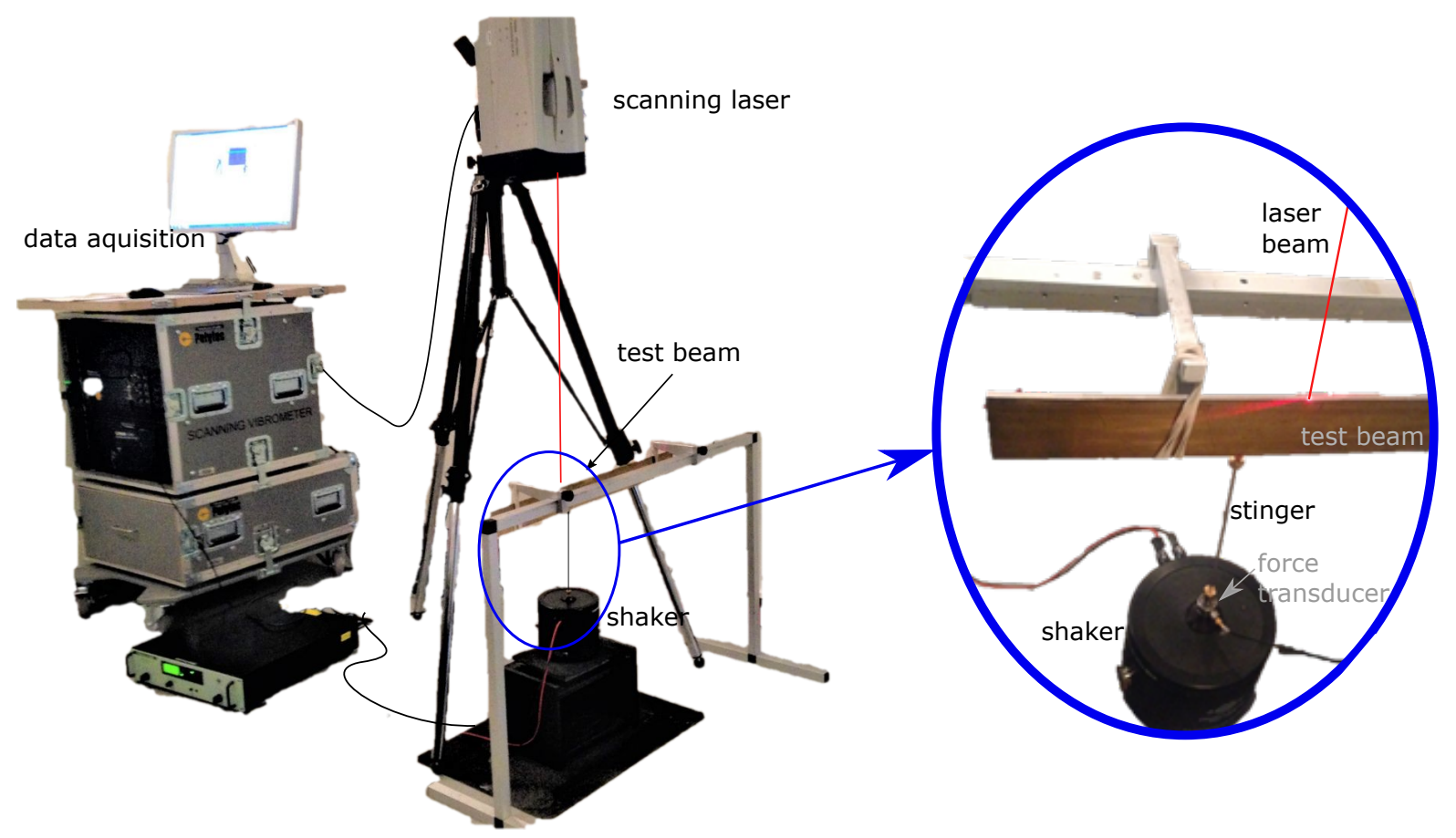

Figure 5: Experimental setup

The beam dimensions $\left(4.02 \times 60 \times 686.5 \mathrm{~mm}^{3}\right)$ and material has been chosen carefully to allow for measuring natural frequencies above the critical frequency (11), which depends on the material property relation $E / \rho$ and the cross-section property $A / I$. For many beams the critical frequency is high up in the ultrasonic frequency range $\left(f_{\mathrm{c}} \gg 20 \mathrm{kHz}\right)$, which is above 
most relevant mechanical vibrations. Thus, to be able to excite and measure vibrations above the critical frequency a brass beam was chosen $\left(E=91.27 \mathrm{GPa}, \rho=8427 \mathrm{~kg} / \mathrm{m}^{3}\right)$ - which has a lower $E / \rho$ ratio than steel and aluminium (wave speed $\sqrt{E / \rho}$ about $35 \%$ lower). Young's modulus has been estimated by least squares fitting to the first 8 natural frequencies. By (11) the critical frequency is then $17.04 \mathrm{kHz}$, which is well within the limit of the measurement equipment (around $22 \mathrm{kHz}$ ). As appear from Figure 5 the direction of measurement is in the stiff bending direction. The SLDV scans over the length of the beam in 160 points, though without measurements at the elastic bands, as these block the lasers view of the beam. When post-processing, the few missing points are interpolated and obvious outliers are removed. Each measurement point on the beam, along with the measured input force, provides a frequency response function. Data retrieved in the PSV-software is exported to the modal analysis software ME'Scope where the mode shapes are extracted.

Pretests were made to validate the experimental setup: The brass beam was hammer impacted and the lowest 14 natural frequencies estimated by using frequency responses between measured input force and accelerometer output. The frequencies were compared to those obtained with shaker excitation and SLDV measurements; the deviations were less than $0.5 \%$ for almost all frequencies, (only two frequencies deviated more than $1 \%$ ). To check that the mode shapes obtained above the critical frequency are not too affected by limits in the measurement equipment, a steel beam, with a critical frequency of $161.12 \mathrm{kHz}$ was tested. With the measurable frequency range only up to $22 \mathrm{kHz}$, the obtained modes are much below the critical frequency. In this case it was possible to measure all the lowest 26 modes and obtain a diagonal MAC-value matrix with all values exceeding 0.95 ; this shows that the measured mode shapes follow the theoretical modes well (cf. Section 2.3). Furthermore, the deviation between TBT and measured frequencies was only $1.4 \%$ for the 26th natural frequency and lower for the other frequencies. These pretests increase the confidence in the results obtained for the brass beam setup. A numerical 3D COMSOL-model of the beam was made to have an extra comparison to TBT and experimental results, bypassing $1 \mathrm{D}$ beam theory as well as measurement inaccuracies.

\section{Results}

\subsection{Frequency responses}

Figure 6 shows a typical frequency response measured with the SLDV at a single point on the beam. Below the critical frequency (dashed vertical line in lower panel) there are mainly frequency peaks from beam bending. Above the critical frequency there are many frequency peaks, as this range features natural frequencies for both transverse planes, as well as torsional, longitudinal and plate like modes. As the purpose is to validate TBT, the theory cannot be used to identify the peaks above the critical frequency, as it is not known which peak belongs to which type of mode. The mode shapes should be used instead. Consequently, this section identifies the mode shapes corresponding to transverse bending in the plane of interest first, and then compares the measured frequencies of those modes with TBT, and with 3D COMSOL model predictions. 

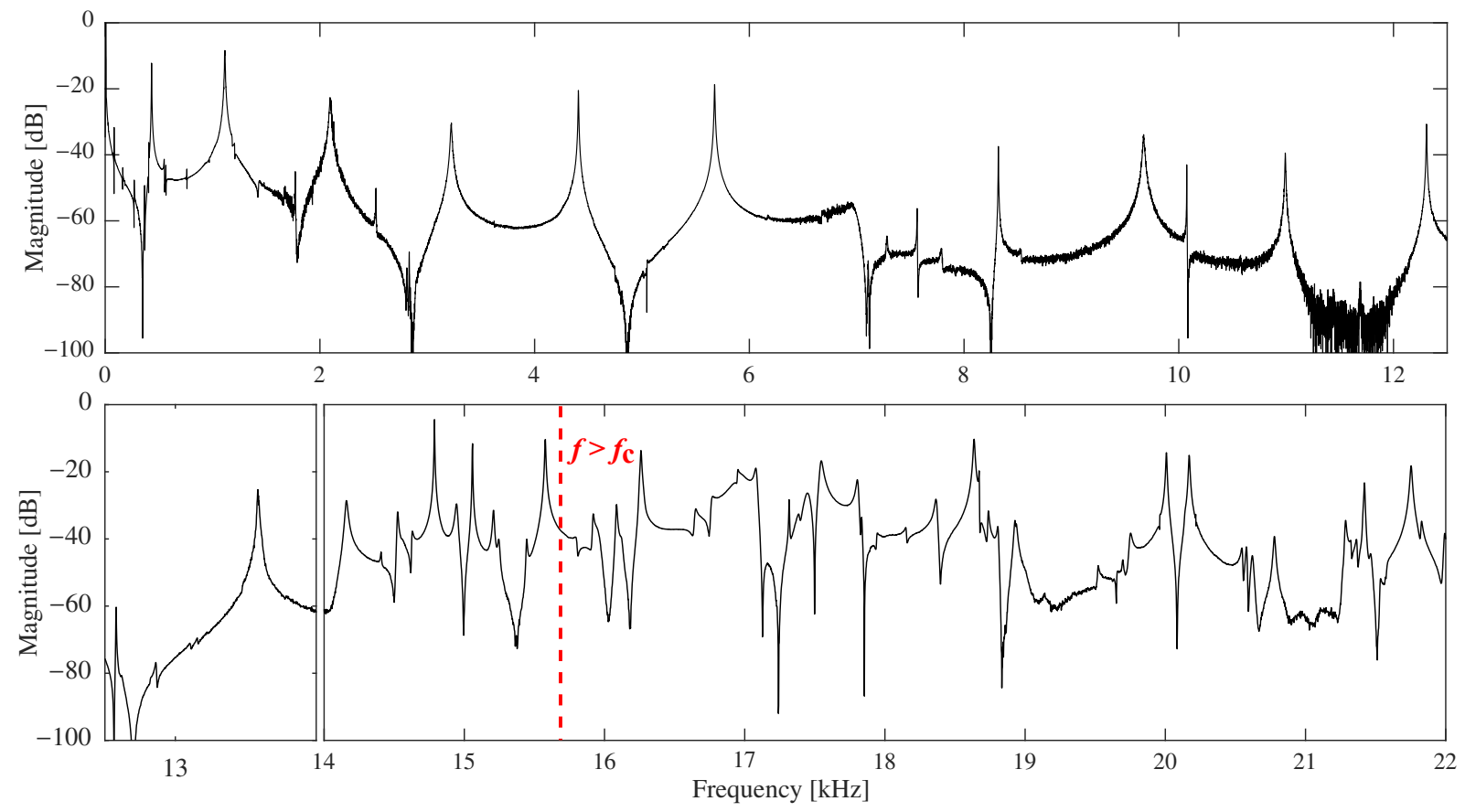

Figure 6: Example frequency response for experimental brass beam. Upper panel: 0-12.5 kHz; lower panels: $12.5-22 \mathrm{kHz}$; dashed vertical line: approximate experimental critical frequency $f_{\mathrm{c}}$. (for technical reasons the three panels are from different samples).

\subsection{Mode shapes}

Figure 7 shows the normalized experimental mode shapes $\varphi_{\text {exp }}$ set up against the theoretical TBT modes $\varphi_{\text {TВT }}$. Experimental modes 1-14 are below $f_{\mathrm{c}}$, and appear very alike TBT modes. The supercritical modes 15-24 also qualitatively show agreement with TBT predictions. Modes 16 and 18 are, unlike the other modes, rather irregular. This is due to other modes that coincide with these transverse bending modes: The ME'Scope software makes a fit, expecting a single peak, it cannot handle if two peaks are intermixed. Mode 16 is close to a bending mode in the other plane, and mode 18 to a plate bending mode (this both shows from the measurements and from the 3D COMSOL model predictions). 
\#1, $0.433 \mathrm{kHz}$

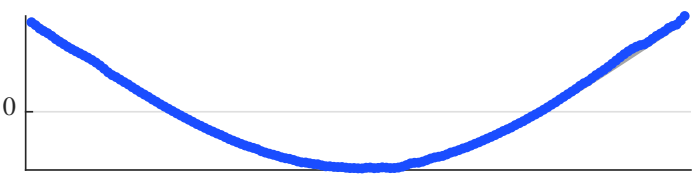

\#3, $2.106 \mathrm{kHz}$

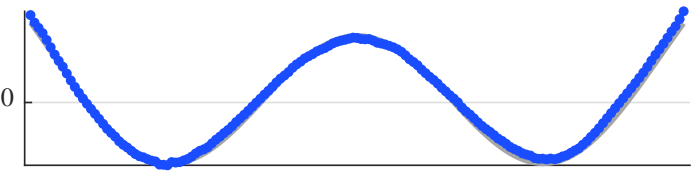

\#5, $4.409 \mathrm{kHz}$

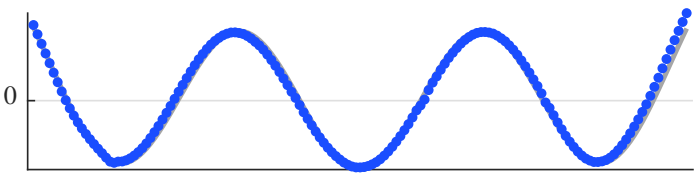

\#7, $6.969 \mathrm{kHz}$

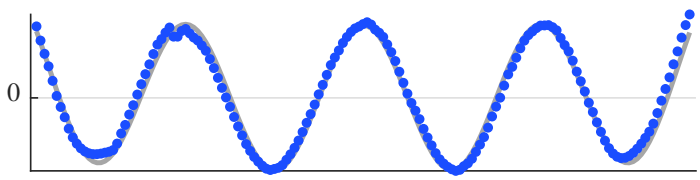

\#9, $9.674 \mathrm{kHz}$

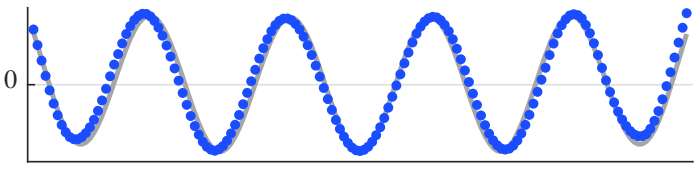

\#11, $12.308 \mathrm{kHz}$

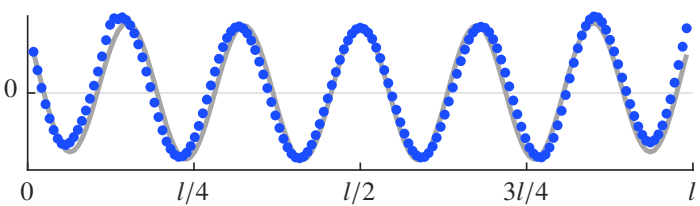

\#2, $1.116 \mathrm{kHz}$

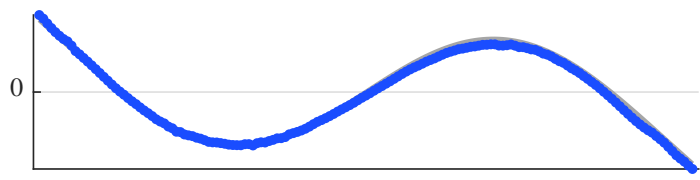

\#4, $3.225 \mathrm{kHz}$

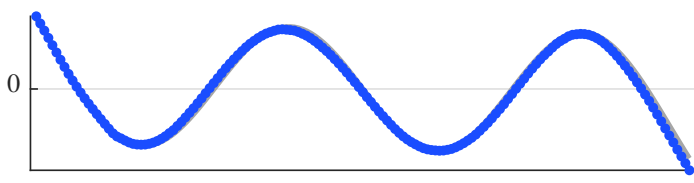

\#6, $5.677 \mathrm{kHz}$

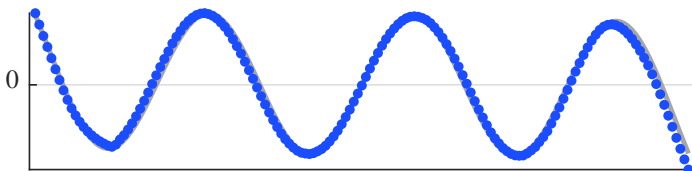

\#8, $8.320 \mathrm{kHz}$

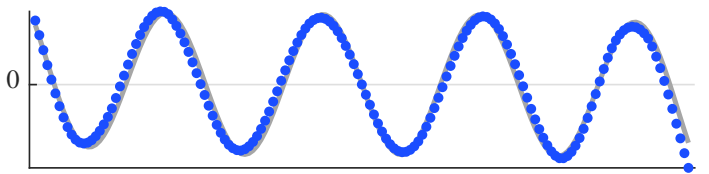

\#10, $10.993 \mathrm{kHz}$

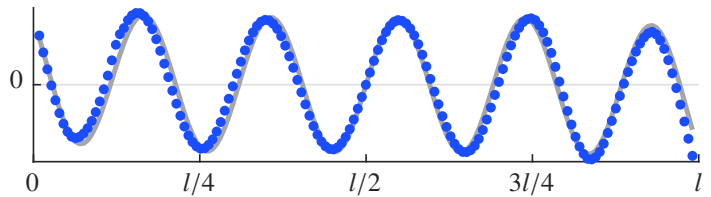

\#12, $13.588 \mathrm{kHz}$

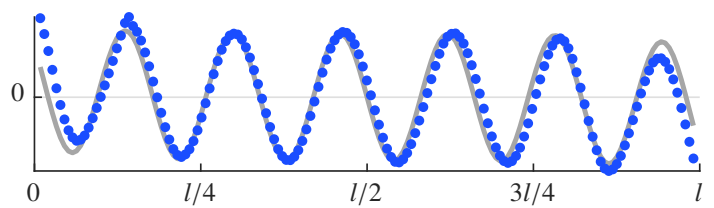

Figure 7: Free-free brass beam mode shapes. Solid line: theoretic TBT modes $\varphi_{\mathrm{TBT}}$; dots: experimentally measured modes $\varphi_{\exp }$. (Figure continues in next panel.) 


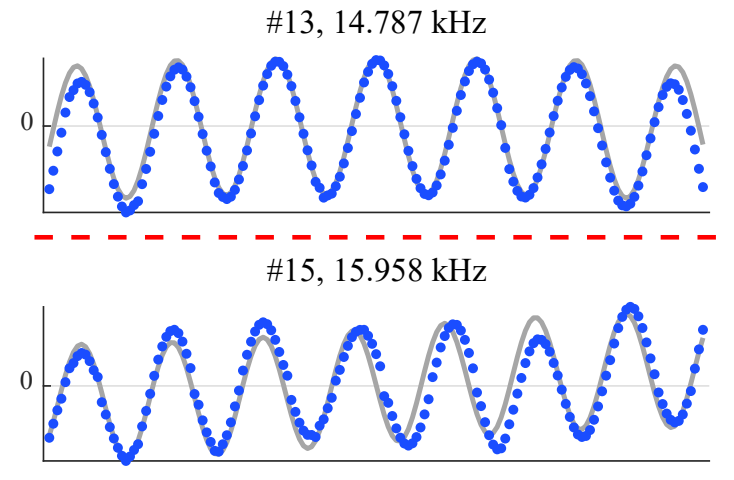

$\# 17,17.317 \mathrm{kHz}$

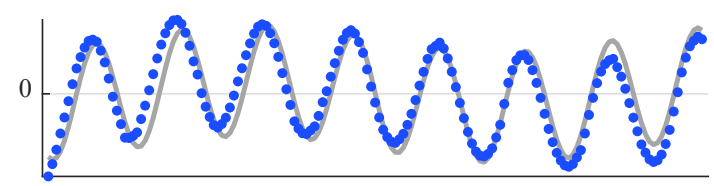

\#19, $18.636 \mathrm{kHz}$

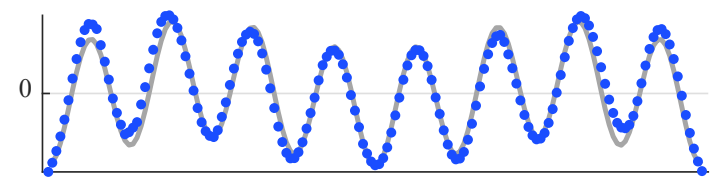

$\# 21,20.008 \mathrm{kHz}$

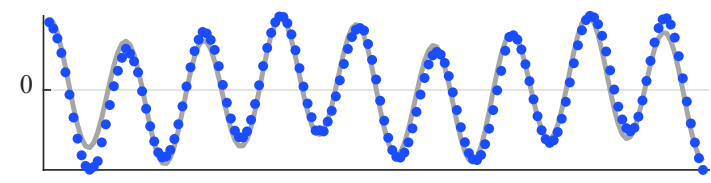

\#23, $21.420 \mathrm{kHz}$

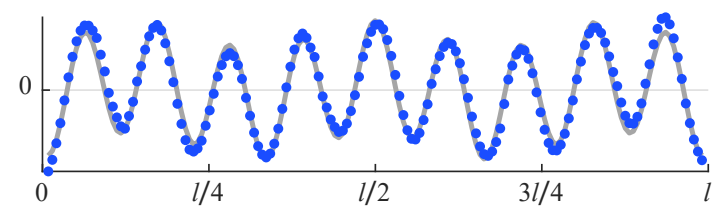

\#14, $15.577 \mathrm{kHz}$
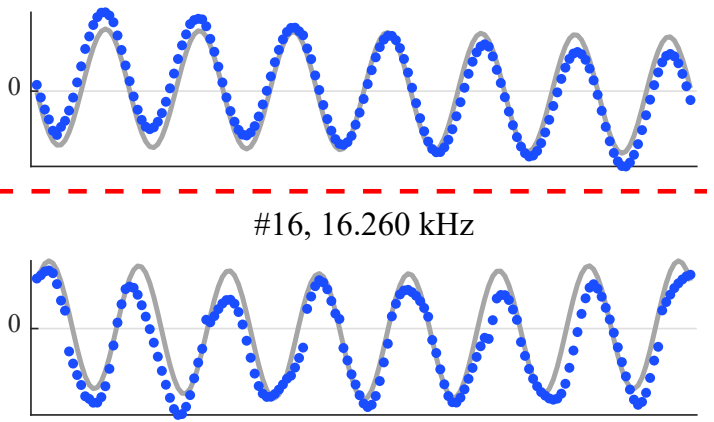

$\# 18,17.544 \mathrm{kHz}$

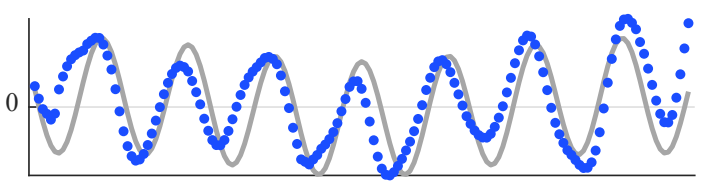

$\# 20,18.672 \mathrm{kHz}$

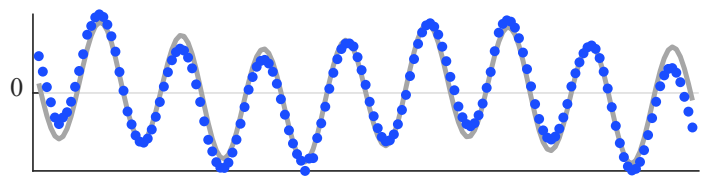

$\# 22,20.170 \mathrm{kHz}$
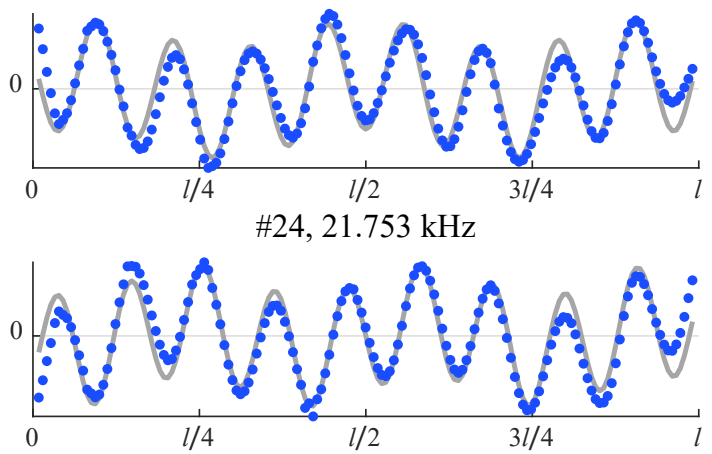

Figure 7: Continued.

Figure 8(a) shows the MAC-matrix between the experimental mode shapes and TBT mode shapes. As a reference for comparison Figure 8(b) shows the 'ideal' MAC-matrix for TBT modes against TBT modes with the experimental mode shape order (which is why it is not identical to Figure 4). Both figures have the same pattern: a diagonal up to the critical frequency, and thereafter a more complicated correlation between the modes. There is the same number of measurement points for all modes, for which reason the wavelength resolution decrease the higher the mode (fewer point per wavelength). This also means that if a single measurement point is off, it will appear more strongly so (i.e. off) in the MAC-value for higher modes. The diagonal MAC-values of the supercritical modes are all above 0.85 , and most of them above 0.9, with the exception of mode 16 and 18. Figure 8 (c) shows the difference between Figure 8 (a) and Figure 8(b) (dark color indicating large difference, white no difference). It shows that not only is the diagonal close to theory, but the chess pattern is also in agreement. Again it shows that it is only mode 16 and 18 that show significant disagreement, which, as stated previously, can be explained by coexistence of other plane modes. 


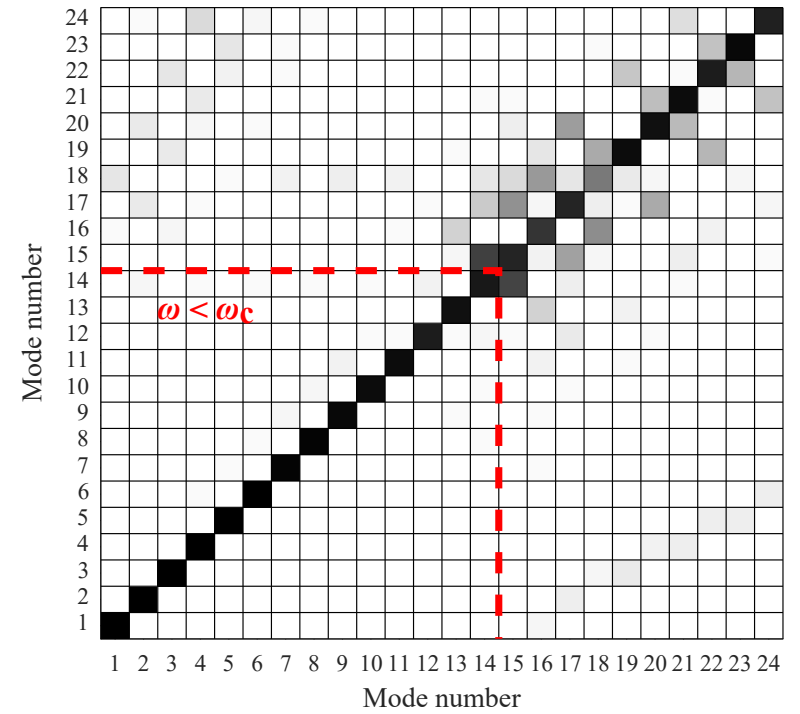

(a)

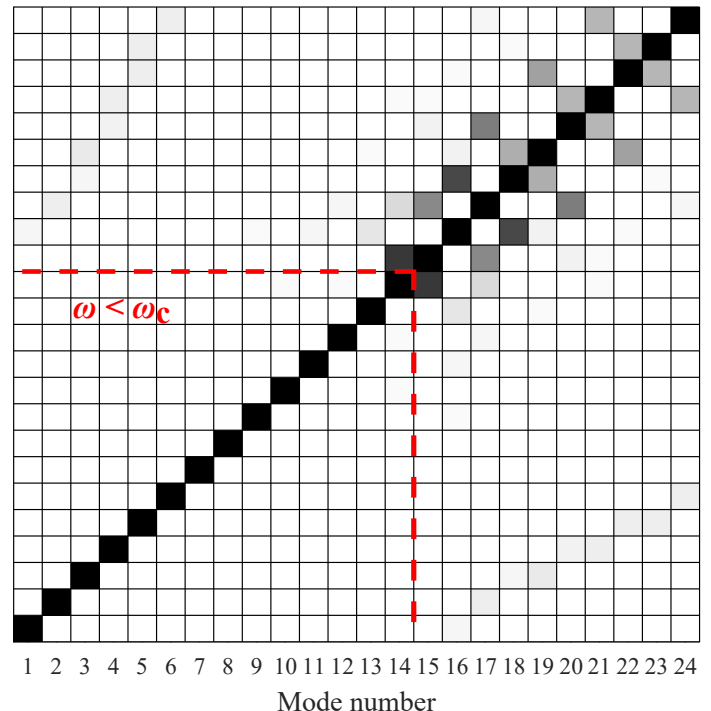

(b)

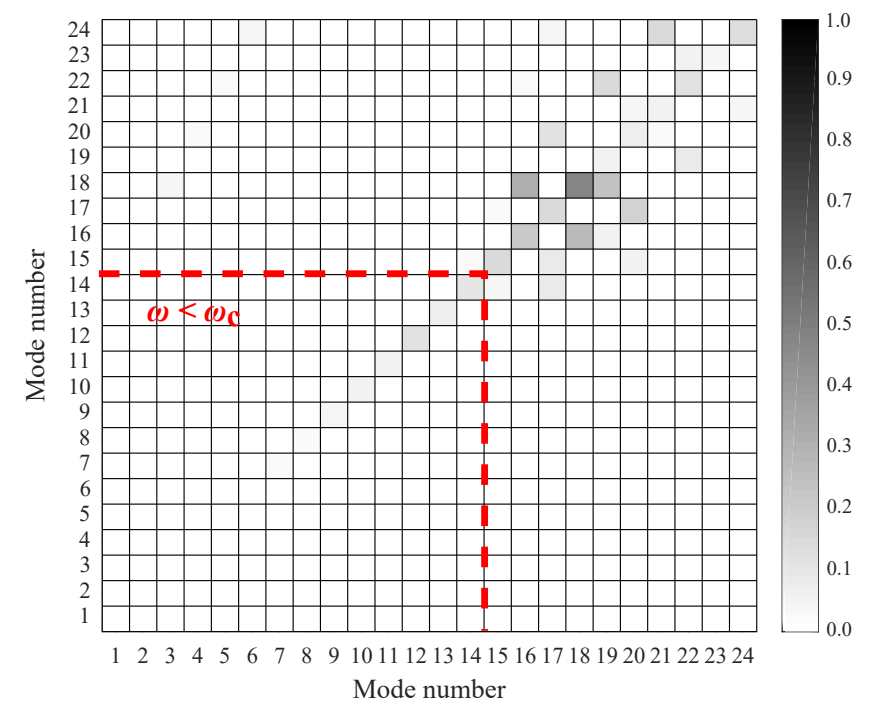

(c)

Figure 8: MAC matrices and differences: (a) $\varphi_{\mathrm{exp}}$ versus $\varphi_{\mathrm{TBT}}$; (b) $\varphi_{\mathrm{TBT}}$ versus $\varphi_{\mathrm{TBT}}$; (c) Difference between (a) and (b).

\subsection{Natural frequencies}

Table 1 shows the experimentally measured natural frequencies $f_{\text {exp }}$, identified by the mode shape analysis in Section 4.2, along with comparisons to Timoshenko predictions $f_{\mathrm{TBT}}$ and numerical COMSOL predictions $f_{\mathrm{COM}}$. The theoretical critical frequency is $17.04 \mathrm{kHz}$, however experimentally it is lower, between [15.6-15.9] kHz (between mode 14 and 15). The measured supercritical natural frequencies come in close pairs, as predicted by TBT and COMSOL. The deviations to $f_{\mathrm{TBT}}$ and $f_{\mathrm{COM}}$ increase with the mode number. The modes close to the critical frequency deviate the most (up to $8.4 \%$ ), but well past the critical frequency the deviations stop increasing and settles at approximately $5 \%$. The $f_{\mathrm{TBT}}$ and $f_{\mathrm{COM}}$ predictions are less than $1 \%$ different in most cases. In Section 4.2 each experimental mode shape has been matched with a theoretical mode shape, without considering how well the frequency match. This reveals that in this case, the experimental mode shape order is not exactly the same as TBT and COMSOL 
predicts. As mentioned in Section 2.1 the order of an odd/even pair is very sensitive to changes in parameters as the two frequencies in a pair are very close together in the frequency spectrum. For this TBT prediction meaningful physical parameters were used giving two swapped pairs. E.g. TBT predicts $18.302 \mathrm{kHz}$ to be an odd mode and $18.508 \mathrm{kHz}$ to be an even mode, in the experiment $17.317 \mathrm{kHz}$ is odd (mode 17) and $17.544 \mathrm{kHz}$ is even (mode 18), thus odd and even in that pair are swapped. The same apply to mode pair 19-20 for TBT, and for COMSOL for mode pair 17-18 (swapped order is indicated by an asterisk in Table 1).

Table 1: Experimental natural frequencies $f_{\text {exp }}$ compared to TBT and numerical 3D COMSOL values. $\Delta$ : relative deviation; $f<f_{\mathrm{c}}$ for mode $1-14 ; *$ : mode order as matched in Section 4.2 .

\begin{tabular}{lrrrrrrr}
\hline Mode & even/odd & $\begin{array}{r}f_{\text {exp }} \\
\mathrm{kHz}\end{array}$ & $\begin{array}{r}f_{\text {TBT }} \\
\mathrm{kHz}\end{array}$ & $\begin{array}{r}f_{\text {COM }} \\
\mathrm{kHz}\end{array}$ & $\begin{array}{r}\Delta_{\text {exp-TBT }} \\
\%\end{array}$ & $\begin{array}{r}\Delta_{\text {exp-COM }} \\
\%\end{array}$ & $\begin{array}{r}\Delta_{\text {СOM-TBT }} \\
\%\end{array}$ \\
\hline 1 & $\mathrm{E}$ & $\mathbf{0 . 4 3 3}$ & 0.419 & 0.419 & 3.3 & 3.2 & 0.1 \\
2 & $\mathrm{O}$ & $\mathbf{1 . 1 1 6}$ & 1.103 & 1.104 & 1.1 & 1.0 & 0.1 \\
3 & $\mathrm{E}$ & $\mathbf{2 . 1 0 6}$ & 2.039 & 2.042 & 3.2 & 3.0 & 0.2 \\
4 & $\mathrm{O}$ & $\mathbf{3 . 2 2 5}$ & 3.150 & 3.159 & 2.3 & 2.1 & 0.3 \\
5 & $\mathrm{E}$ & $\mathbf{4 . 4 0 9}$ & 4.381 & 4.397 & 0.6 & 0.3 & 0.4 \\
6 & $\mathrm{O}$ & $\mathbf{5 . 6 7 7}$ & 5.690 & 5.717 & -0.2 & -0.7 & 0.5 \\
7 & $\mathrm{E}$ & $\mathbf{6 . 9 6 8}$ & 7.049 & 7.089 & -1.2 & -1.7 & 0.6 \\
8 & $\mathrm{O}$ & $\mathbf{8 . 3 2 0}$ & 8.438 & 8.493 & -1.4 & -2.1 & 0.7 \\
9 & $\mathrm{E}$ & $\mathbf{9 . 6 7 4}$ & 9.842 & 9.914 & -1.7 & -2.5 & 0.7 \\
10 & $\mathrm{O}$ & $\mathbf{1 0 . 9 9 3}$ & 11.249 & 11.339 & -2.3 & -3.1 & 0.8 \\
11 & $\mathrm{E}$ & $\mathbf{1 2 . 3 0 8}$ & 12.650 & 12.757 & -2.8 & -3.6 & 0.8 \\
12 & $\mathrm{O}$ & $\mathbf{1 3 . 5 8 8}$ & 14.033 & 14.153 & -3.3 & -4.2 & 0.8 \\
13 & $\mathrm{E}$ & $\mathbf{1 4 . 7 8 7}$ & 15.375 & 15.497 & -4.0 & -4.8 & 0.8 \\
$14 f<f_{\mathrm{c}} \mathrm{O}$ & $\mathbf{1 5 . 5 7 7}$ & 16.609 & 16.644 & -6.6 & -6.8 & 0.2 \\
\hline 15 & $\mathrm{O}$ & $\mathbf{1 5 . 9 5 8}$ & 17.303 & 17.076 & -8.4 & -7.0 & -1.3 \\
16 & $\mathrm{E}$ & $\mathbf{1 6 . 2 6 0}$ & 17.519 & 17.355 & -7.7 & -6.7 & -0.9 \\
\hline 17 & $\mathrm{O}$ & $\mathbf{1 7 . 3 1 7}$ & $* 18.508$ & $* 18.322$ & -6.9 & -5.8 & -1.0 \\
18 & $\mathrm{E}$ & $\mathbf{1 7 . 5 4 4}$ & $* 18.302$ & $* 18.229$ & -4.3 & -3.9 & -0.4 \\
\hline 19 & $\mathrm{E}$ & $\mathbf{1 8 . 6 3 6}$ & $* 19.726$ & 19.558 & -5.8 & -4.9 & -0.9 \\
20 & $\mathrm{O}$ & $\mathbf{1 8 . 6 7 2}$ & $* 19.657$ & 19.564 & -5.3 & -4.8 & -0.5 \\
\hline 21 & $\mathrm{O}$ & $\mathbf{2 0 . 0 0 8}$ & 21.093 & 20.945 & -5.4 & -4.7 & -0.7 \\
22 & $\mathrm{E}$ & $\mathbf{2 0 . 1 7 0}$ & 21.123 & 20.978 & -4.7 & -4.0 & -0.7 \\
\hline 23 & $\mathrm{E}$ & $\mathbf{2 1 . 4 2 0}$ & 22.540 & 22.397 & -5.2 & -4.6 & -0.6 \\
24 & $\mathrm{O}$ & $\mathbf{2 1 . 7 5 3}$ & 22.666 & 22.461 & -4.2 & -3.3 & -0.9 \\
\hline & & & & & & & \\
\hline
\end{tabular}




\section{Discussion}

This discussion is sectioned to separate different aspects of interest, all relating to the works mentioned in the introduction [3, 4, 6, 7, 1]. As distinction between modal properties for various boundary conditions is necessary, these points of discussion only regard free-free boundary conditions. The distinction between different boundary conditions revolves firstly around the question of whether or not the frequency equation factorizes, which it does not for a majority of boundary conditions [5] (e.g. free-free, clamped-clamped or clamped-free), but does for hinged-hinged, hinged-guided, guided-guided [4]. In cases where the frequency equation factorizes, the fundamental difference from the free-free case is that the mode shapes are not affected by the critical frequency. Because of this significant difference between some boundary conditions we here limit conclusions to the free-free case. Aspects of concern are: the existence of supercritical frequency pairs and mode shapes; the value of the shear coefficient; the importance of mode shapes and proper mode matching; possible interference of other plane modes, comparison to a modified TBT model; and views on the usefulness of TBT for supercritical predictions, compared to other modelling options.

\subsection{The existence of supercritical frequency pairs and their mode shapes}

First it must be emphasized that the experimental results presented in this work show an excellent agreement between the TBT predicted mode shapes and those measured in the laboratory (most MAC-values in the diagonal exceeds 0.9 ). The measured natural frequencies deviate from TBT predictions in the order of $5 \%$ above the critical frequency (cf. Section 4). These results are at least the fourth time supercritical frequency pairs has been reported [3, 4, 6, 7]: This collection of experiments have been conducted on beams with very different dimensions, materials and different types of measurement equipment and with such a convincing documentation the pairs cannot be disregarded. However, it can be argued if the frequency predictions are accurate enough to be useful, this is discussed in Section 5.6.

\subsection{Shear coefficient value - does the error increase or stabilize for supercritical modes?}

Around the critical frequency, the frequency deviations in the experiments are bigger than for lower modes, but the error stabilizes at approximately $5 \%$ above the critical frequency. If it was possible to measure even higher frequencies it could be established if the deviations increase further or if they stay on a constant level. A stable error could indicate a bias in the model, e.g. as suggested by [17] the shear coefficient $k$ might assume another value above $f_{\mathrm{c}}$. If the shear coefficient $k$ is changed, according to one of the modifications in [17], the error in TBT predictions in Table 1 can at least be reduced to an order of $3 \%$.

\subsection{The order of mode shapes and importance of mode matching}

In Section 4 it was found that a strict system for sorting and matching of modes was necessary to ensure that what is compared is actually comparable. Here the MAC tool showed useful for quantifying the correlation between theoretical and experimental mode shapes, which is of utmost importance when afterwards making a comparison between the frequencies. The results showed that in some cases the predicted mode shape order was different from that observed in the experiment. Had natural frequencies alone been considered it would falsely have given a smaller error between TBT and experiment than was actually the case, leaving a meaningless comparison. Thus, the MAC tool provides confidence in the comparisons made. 


\subsection{The interference of other plane modes - how to distinguish}

There are other reasons for the mode shapes to be very important: When considering very high modes a beam is no longer just a beam. It is a 3D structure. It can bend in both transverse planes, deform like a plate, and bend longitudinally, show complex torsional behaviour, or even some combination of all this. The multitude of modes can easily be shown by employing a numerical model in e.g. COMSOL, ANSYS etc. All these modes exists in close succession in the frequency spectrum, why again it is not enough to measure frequencies to validate TBT; consideration of the mode shapes must be included.

In [5] the conclusion was to disregard the prediction above $f_{\mathrm{c}}$ due to large deviations to experimental results. However, to provide more insights a new numerical COMSOL model has been made, with the dimensions and physical properties given in [5]. This reveals that quite possibly one of the recorded experimental frequency pairs really involves a bending mode around the longitudinal axis combined with transverse bending, (the beam is only 4 times longer than its' width). Theoretically, the first bending mode around the longitudinal axis is about $200 \mathrm{kHz}$, and the block is almost symmetric, potentially causing modal interaction. If that frequency is excluded, the errors for the highest modes decrease from the reported $27 \%$ to $11 \%$. Overall the new COMSOL model shows that considering the block's modes as beam bending at frequencies that high is too simplified. Thus even if TBT theory in principle predicts the supercritical pairs and motion, it is still limited to describing beam bending modes.

In [6, 7] the experimentally measured supercritical natural frequencies are found to deviate less than $1 \%$ from TBT, measured in the stiff bending plane of the beam, and the experimentally measured mode shapes have the same number of half wavelengths as TBT predicts. However, in Figure 7 it is seen that the height of the supercritical mode shape peaks are different along the beam length (a product of the shear/rotation relation from Section 2.2. This feature is easily missed if the mode shapes are only plotted atop each other. For bending mode shapes below $f_{\mathrm{c}}$ there is not this variation in peak height. A reproduction of the beam in COMSOL showed that there are mode shapes which are a combination of torsion and bending in the most flexible plane that has natural frequencies below $f_{\mathrm{c}}$ with the same number of half wavelengths as that of the stiff direction, but not the varying peak height, with frequencies quite close to the supercritical in the stiff bending plane. Therefore, without a quantitative comparison it cannot be eliminated if some of the measured modes, leading to such small deviations in frequency, are torsion-flexible bending modes. The MAC tool could reject or confirm this.

\subsection{Modifying the model to avoid a critical frequency}

In [1] a modified version of TBT is suggested. The difference lies in the associated rotational inertia forces that are set to $\rho I^{\prime}{ }^{\prime}$ (i.e. not based on inertial coordinates) and not $\rho I \ddot{\Psi}$. This change removes the prediction of a critical frequency and thus also supercritical frequency pairs. The argument is that the TBT supercritical predictions are inaccurate and yields more than necessary. If however, the modified theory is attempted matched with the modes and frequencies measured above the critical frequency reported in Section 4 they do not match; the mode shapes do not fit any of the pairs measured. In [1] it is not suggested to fit above $f_{\mathrm{c}}$, but why modify a model equation to be less inclusive than the existing? An advantage could be simplicity, but for freefree boundary conditions the equations to solve for the modified theory are the same.

\subsection{Better alternatives? - Requirements for a validation of supercritical TBT predictions}

TBT refers to a $1 \mathrm{D}$ beam model. It cannot capture the full behaviour of three dimensional high-frequency motion in a structure. It approximates as the Bernoulli-Euler model. However, 
unlike Bernoulli-Euler the TBT theory actually predicts very accurately up until the critical frequency, and overall it also captures the fundamental change in behaviour when passing over to supercritical modes, i.e. the occurrence of frequency pairs, and a new, seemingly erratic, order of modes (odd-even interchanges), as well as the complex composition of shear and rotation (cf. Section 2.2).

Returning to the experiment reported in [5] the test object has dimensions $10 \times 10 \times 40 \mathrm{~mm}^{3}$. The difference of $10 \%$ between TBT and experiment occurs at mode 9 and 10 at frequencies of $221 \mathrm{kHz}$, which is $28 \%$ larger than the critical frequency $159 \mathrm{kHz}$. Considering this, it could actually be perceived incredible that the TBT prediction is still that accurate.

For the present study, Table 1 shows that for the brass beam a 3D model is not superior. So, there is not actually a better alternative to TBT for describing supercritical frequencies and mode shapes. In other cases it could be found that an 3D model is clearly superior; it depends on the beam's thickness to length ratio and the frequency range of interest. The brass beam is only $4.02 \mathrm{~mm}$ thick (and $686.5 \mathrm{~mm}$ long), it cannot have significant bending in that plane, why a numerical 3D model and TBT predict the same frequencies. If the thickness was twenty times larger, TBT still predicts the same frequencies, whereas the 3D model predictions changes.

If interest is in the supercritical mode shapes, the TBT theory is excellent, as far up the frequency spectrum as it makes sense to consider the 3D structure a beam. If interest is in the natural frequencies, there is an error in the order of $5 \%$, but since a 3D model gives the same, there is really no better alternative.

\section{Conclusions}

A SLDV was used for measuring mode shapes above the Timoshenko-critical frequency $f_{\mathrm{c}}$ of a free-free beam, with the purpose of investigating the accuracy of Timoshenko beam theory predictions. The experiments with the brass beam revealed frequency pairs in the supercritical domain. This has now been reported several times, and these pairs can be considered a fact. Above $f_{\mathrm{c}}$ the deviations between TBT and experimental natural frequencies are within $5 \%$, larger than below $f_{\mathrm{c}}$. The MAC-value tool has been used to pair experimental modes with theoretic, showing that the TBT predicted modes are very accurate in comparison with the experimentally measured modes. The experiment also showed how the mode shape order (odd and even) swaps back and forth above the critical frequency, as TBT and 3D models also predict, but also that the order predicted by TBT and the 3D model is not consistent with experimental observations. Comparison to 3D models showed that other plane modes can easily be misinterpreted as bending modes. The MAC tool revealed the need for proper sorting and ordering of modes, and emphasized how both theoretical natural frequencies and mode shapes must coincide with the experimental results for the experiment to be considered a validation of Timoshenko theory. Returning to the three debate positions mentioned in the Introduction, this present contribution shows that TBT should not be disregarded above the critical frequency, at least for free-free boundary conditions. Future work could include general boundary conditions to determine if TBT is generally valid above $f_{c}$. TBT is still only a one-dimensional beam model; thus it should be applied with significant care for predicting supercritical frequencies, considering if the structure can meaningfully be modelled as a beam for that high frequencies. 


\section{References}

[1] I. Elishakoff, J. Kaplunov, E. Nolde, Celebrating the Centenary of Timoshenko's Study of Effects of Shear Deformation and Rotary Inertia, Applied Mechanics Reviews 67 (6) (2015) 060802. doi:10.1115/1. 4031965.

[2] S. Timoshenko, On the correction for shear of the differential equation for transverse vibrations of prismatic bars, Philosophical Magazine 41 (245).

[3] K. T. Chan, X. Q. Wang, R. M. C. So, S. R. Reid, Superposed Standing Waves in a Timoshenko Beam, Proceedings: Mathematical, Physical and Engineering Sciences 458 (2017) (2002) 83-108. doi:10.2307/ 3067421.

[4] N. G. Stephen, The second spectrum of Timoshenko beam theory - Further assessment, Journal of Sound and Vibration 292 (1-2) (2006) 372-389. doi:10.1016/j.jsv.2005.08.003

[5] N. G. Stephen, S. Puchegger, On the valid frequency range of Timoshenko beam theory, Journal of Sound and Vibration 297 (3-5) (2006) 1082-1087. doi:10.1016/j.jsv.2006.04.020.

[6] A. Díaz-De-Anda, J. Flores, L. Gutiérrez, R. A. Méndez-Sánchez, G. Monsivais, A. Morales, Experimental study of the Timoshenko beam theory predictions, Journal of Sound and Vibration 331 (26) (2012) 57325744. doi:10.1016/j.jsv.2012.07.041.

[7] G. Monsivais, A. Díaz-de Anda, J. Flores, L. Gutiérrez, A. Morales, Experimental study of the Timoshenko beam theory predictions: Further results, Journal of Sound and Vibration 375 (2016) 187-199. doi:10. $1016 / j \cdot j \mathrm{jv} .2016 .04 .003$.

[8] B. Abbas, J. Thomas, The second frequency spectrum of Timoshenko beams, Journal of Sound and Vibration 51 (1) (1977) 123-137. doi:10.1016/0022-460X (81)90520-4.

[9] S. Ekwaro-Osire, D. H. S. Maithripala, J. M. Berg, a Series Expansion Approach To Interpreting the Spectra of the Timoshenko Beam, Journal of Sound and Vibration 240 (4) (2001) 667-678. doi:10.1006/jsvi. 2000.3253

[10] M. Levinson, D. Cooke, On the two frequency spectra of Timoshenko beams, Journal of Sound and Vibration 84 (3) (1982) 319-326. doi:10.1016/0022-460X (82)90480-1.

[11] W. Flügge, Handbook of Engineering Mechanics, McGraw-Hill, New York, 1962. doi:10.1115/1. 3630093

[12] G. Bhashyam, G. Prathap, the Second Frequency Spectrum Beams of, Journal of Sound and Vibration 76 (3) (1981) 407-420.

[13] W. Howson, A. Watson, On the provenance of hinged-hinged frequencies in Timoshenko beam theory, Computers \& Structures 197 (2018) 71-81. doi:10.1016/j.compstruc. 2017.11.017

[14] J. C. Torres-Guzmán, A. Díaz-de Anda, J. A. Otero, A. Morales, L. Gutiérrez, G. Monsivais, J. Flores, On the warping of the extreme ends of a beam under flexural oscillations, Journal of Sound and Vibration 435 (2018) 234-245. doi:10.1016/j.jsv.2018.08.019

[15] D. Ewins, Modal testing : Theory and Practice, Research Studies Press, 2000.

[16] R. J. Allemang, The modal assurance criterion - Twenty years of use and abuse, Sound and Vibration 37 (8) (2003) 14-21. doi:10.1016/j.chemgeo.2006.02.014.

[17] J. A. Franco-Villafañe, R. A. Méndez-Sánchez, On the Accuracy of the Timoshenko Beam Theory Above the Critical Frequency: Best Shear Coefficient, Journal of Mechanics (January) (2016) 1-4. arXiv:1405.4885. doi:10.1017/jmech.2015.104. 